\title{
Dependence of Curvature Radii of the Apexes of W Tips on Growth of Au- and Pd-Coated Nanopyramid
}

\author{
Hirotaka Asai, ${ }^{\dagger}$ Takayuki Tanaka, Hidekazu Murata, Eiji Rokuta \\ Faculty of Science and Technology, Meijo University, Shiogamaguchi, Tempaku, Nagoya 468-8502, Japan \\ † Corresponding author:163441501@ccalumni.meijo-u.ac.jp
}

Received: 9 January, 2020, Accepted: 27 April, 2020, Published: 9 May, 2020

Atomic scale tungsten (W) pyramids coated with monolayer films of noble metals can be produced on the apex of a W tip sharpened by means of chemical etching. In this work, we produced Pd- and Au-coated nanopyramids using W tips with different apex curvature radii as a substrate and examined the yield ratios of the nanopyramid formation. When the curvature radius is larger than $15 \mathrm{~nm}$, both Pd- and Au-coated W nanopyramids grow. However, when the radius is less than $15 \mathrm{~nm}$, only Au-coated nanopyramids grow. We propose that the preferential growth for the Au case is associated with previous calculation results demonstrating that the difference in the anisotropy of the surface energy between the $\mathrm{Au} / \mathrm{W}(111)$ and $\mathrm{Au} / \mathrm{W}(211)$ systems is substantial [S. P. Chen, Surf. Sci. 274, L619 (1992)].

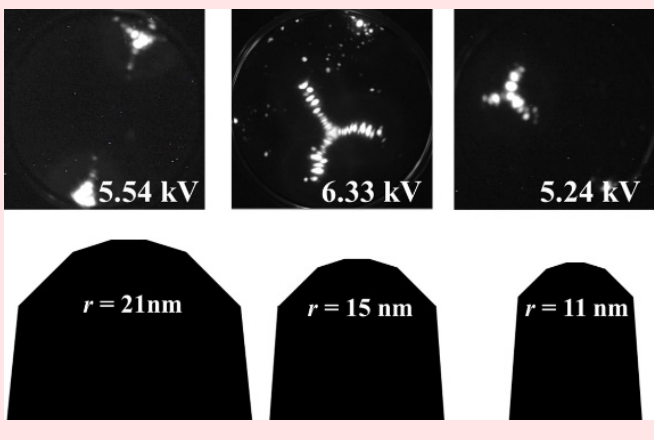

Keywords Nano tip; Field ion microscopy; Faceting; Surface diffusion; Morphology, roughness, and topography

\section{INTRODUCTION}

Attention has been devoted to ultrasharp metal tips for use in electron optic systems as field emitters. Because the sharpness of the metal tip is directly correlated with the spatial coherence of the electron beam, substantial efforts have aimed toward the study of this property. Of all the metal tips reported thus far, the sharpest tip is the singleatom tip, which was first achieved by Fink in 1986 [1]. Following this development, several researchers produced atomically sharp tips using various techniques in the late 1980 s and the early 2000s [2-4]. However, to the best of our knowledge, these tips are not commercially acceptable, primarily because they are not thermodynamically stable. As a potential exception, one compatible field emitter that is more feasible for application is a tungsten (W) pyramid (nanopyramid) coated with a monolayer film of noble metals (NMs). These nanopyramids were first fabricated by $\mathrm{Fu}$ and co-workers, and the field emission characteristics were investigated by Kuo $[5,6]$. Notably, these atomic structures are at thermodynamic equilibrium. Thus, the nanopyramids can be repeatedly formed by annealing at $1000 \mathrm{~K}$ [5].

In a previous study, we demonstrated a spontaneous growth of these nanopyramids by means of a simple method in which $\mathrm{Pd}$ atoms were supplied to the $\mathrm{W}$ tip apex from a NM reservoir, which was analogous to the method applied for the $\mathrm{ZrO} / \mathrm{W}$ Schottky emitter [7, 8]. Although our method has been confirmed as feasible, the yield ratio is still low in our present experiments (approximately 14 successful productions out of 30 trials). We attribute the low yield ratio to the possibility that the necessary conditions for successful production have not yet been established. Thus far, we have focused on the vacuum conditions. However, to further develop the yield ratio, a detailed investigation of the other conditions is needed.

First, the sharpness of the substrate tip must be evaluated. Commonly, the end of the metal tip apex is dependent on the surface component or tip size (i.e., curvature radii), annealing temperature, and electric field [9]. In fact, nanopyramids with three monoatomic-chain ridges cannot grow on NM-coated W tips with large curvature radii $(\mathrm{r}>200 \mathrm{~nm})$ [10-13]. In contrast, NM-coated nanopyramids with monoatomic ridges are generally observed by field ion microscopy (FIM) at low imaging voltages (several to tens of $\mathrm{kV}$ at $\mathrm{Ne}$ ), implying that these substrate $\mathrm{W}$ tips are relatively sharp. It appears that the sharpness of the $\mathrm{W}$ substrate tip is a key parameter governing the nanopyramid growth; however, no 
studies have been reported to confirm the relationship between the nanopyramid growth process and the curvature radius of the $\mathrm{W}$ substrate tip. Therefore, in the present work, we investigated Pd- and Au-coated nanopyramid growth on W tips with different apex curvature radii as a substrate using FIM techniques and examined the yield ratio of nanopyramid formation.

\section{EXPERIMENTAL}

All the experiments were performed in an ultrahigh vacuum chamber equipped with a chevron-type microchannel plate-phosphor screen system for FIM and field emission microscopy (FEM). The base pressure of the chamber was 2 $\times 10^{-8} \mathrm{~Pa}$.

The specimens used in this work were polycrystalline $\mathrm{W}$ wire $(0.25 \mathrm{~mm})$ that was spot-welded to the top of a supportive wire. As a preliminary step, the specimens were chemically treated. The raw $\mathrm{W}$ wires were covered by native oxide and presumably contaminated by hydrocarbons or $\mathrm{Cu}$ particles due to the spot-welding process. To remove the contaminants, the specimens were fully immersed in $2 \mathrm{M}$ $\mathrm{KOH}$ solution and held at a positive voltage for $5 \mathrm{~min}$. As a result, fresh surfaces were obtained for the specimens. Subsequently, we deposited $\mathrm{Au}$ or Pd films as a reservoir on the fresh surface of the $\mathrm{W}$ tips by electroplating. The details of the electroplating process have been reported in Ref. 7. After electroplating, the end of the $\mathrm{W}$ tip was sharpened by traditional electrochemical etching with a $2 \mathrm{M} \mathrm{KOH}$ solution. The top of the $\mathrm{W}$ tip was cleaned and blunted to an arbitrary curvature radius by field evaporation under He-FIM observation. We estimated the curvature radius of the $\mathrm{W}$ substrate tip, $r$, from a helium ion image using the following equation [14]:

$$
r=\frac{n s}{1-\cos \theta}
$$

where $n$ is the mean number of atomic steps from the (110) plane to the center of the $\{211\}$ plane, $s$ is the spacing of the (110) planes, and $\theta$ is the angular separation between the (110) and $\{211\}$ poles. According to Ref. 15, the values of $s$ for tungsten (110) planes and the value of $\cos \theta$ are 0.223 $\mathrm{nm}$ and 0.866 , respectively. Finally, to transfer $\mathrm{Pd}$ or $\mathrm{Au}$ atoms from the reservoir, we annealed the specimen at 1000 $\mathrm{K}$ by resistive Joule heating. To avoid the effect of residual gas on nanopyramid growth, the annealing was carefully performed at a base pressure below $5 \times 10^{-8} \mathrm{~Pa}$.

\section{RESULTS AND DISCUSSION}

First, we demonstrate the structural evolution that occurs for $\mathrm{W}$ tips with different curvature radii. Figure 1 shows the structural evolution of Au-coated W tips with different curvature radii as a function of the annealing treatment time. Images (a), (d), and (h) were obtained using He ions, and the remaining images were obtained using $\mathrm{Ne}$ ions. Images $(\mathrm{a}-\mathrm{c}),(\mathrm{d}-\mathrm{g})$, and $(\mathrm{h}-\mathrm{m})$ present the structural evolution of the $\mathrm{W}$ tips with a curvature radius of 22,17 , and $13 \mathrm{~nm}$, respectively. Typical bcc metal faces, including low-index planes such as $\{110\},\{111\},\{100\}$, and $\{211\}$, were observed, as shown in Figure 1(a, d, h). Following a 30-min annealing treatment of the $\mathrm{W}$ tip at $1000 \mathrm{~K}$, the field ion (FI) pattern drastically changed. The FI patterns converged around the $\{111\}$ plane, indicating that the $\{211\}$ planes were faceted and the $\langle 111\rangle$ pole protruded under vacuum [Figure 1(b, e, i)]. After a 90-min annealing treatment, we found that three linear monoatomic ridges separated neighboring $\{211\}$ planes for the $\mathrm{W}$ tip with a curvature radius of

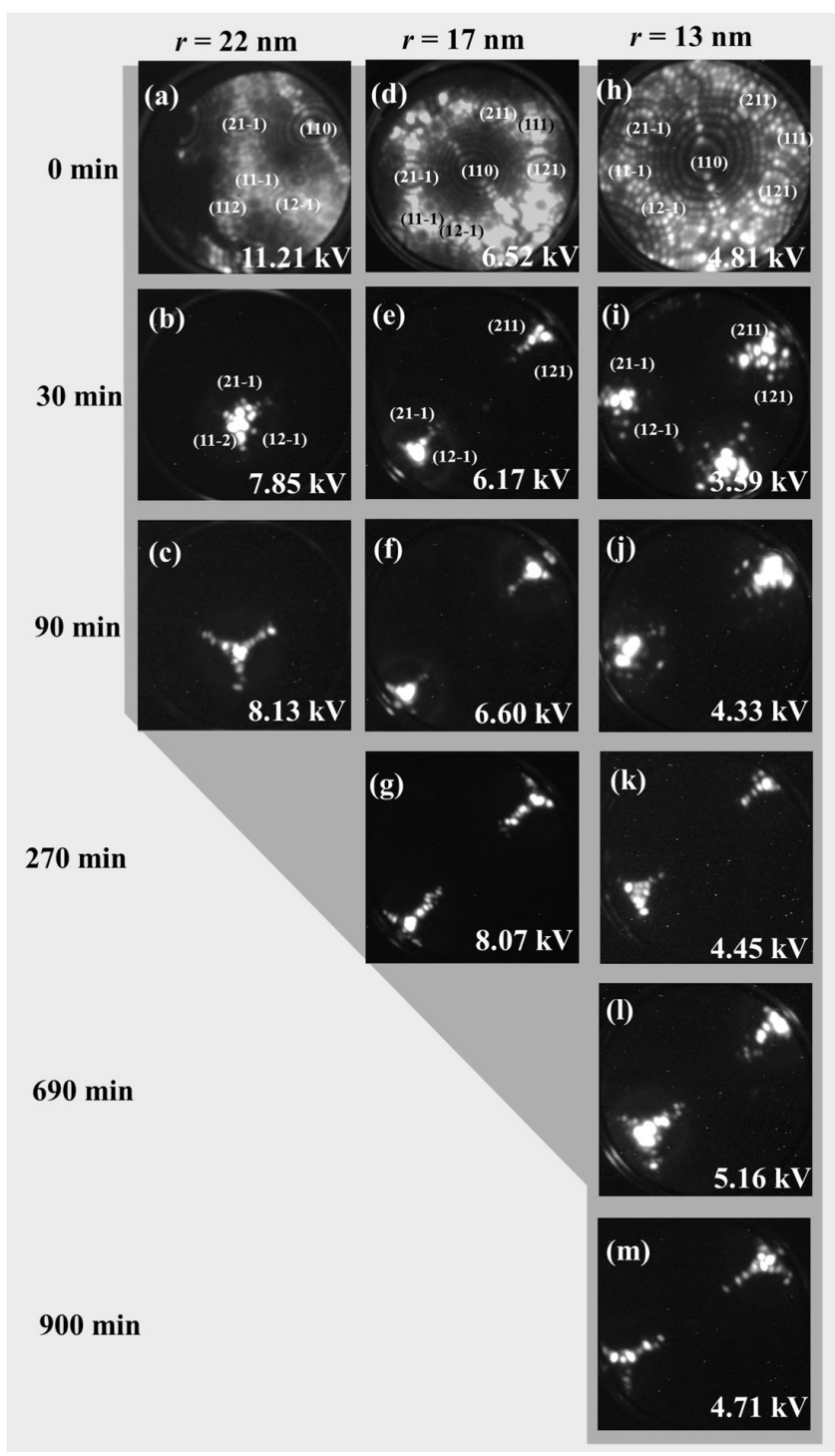

Figure 1: Structural evolution of Au-coated W tips with different curvature radii as a function of annealing time. (a), (d), and (h) show He ion images of a clean $\mathrm{W}$ surface. The remaining images show the structural evolution of the $\mathrm{W}$ tips, obtained by Ne ions. The number in each image denotes the image bias for FIM. The values along the top and left side represent the annealing time and the initial curvature radius, respectively. 
$22 \mathrm{~nm}$ [Figure 1(c)]. This FIM image indicates successful nanopyramid formation. Nanopyramid formation was achieved for $\mathrm{W}$ tips with curvature radii of $17 \mathrm{~nm}$ [Figure $1(\mathrm{~g})$ ] and $13 \mathrm{~nm}$ [Figure $1(\mathrm{~m})$ ] by annealing for 270 and 900 min, respectively. Thus, for the Au-coated W tip, it appears that the nanopyramid formation is independent of the curvature radii for the range examined in this work.

Figure 2 shows the structural evolution of Pd-coated W tips with different curvature radii as a function of the total annealing time. Images (a), (f), and (j) were obtained using $\mathrm{He}$ ions, and the remaining images were obtained using $\mathrm{Ne}$ ions. The left column in Figure 2 presents a series of FIM images depicting the growth of a Pd-coated W tip with a curvature radius of $21 \mathrm{~nm}$; the middle and right columns

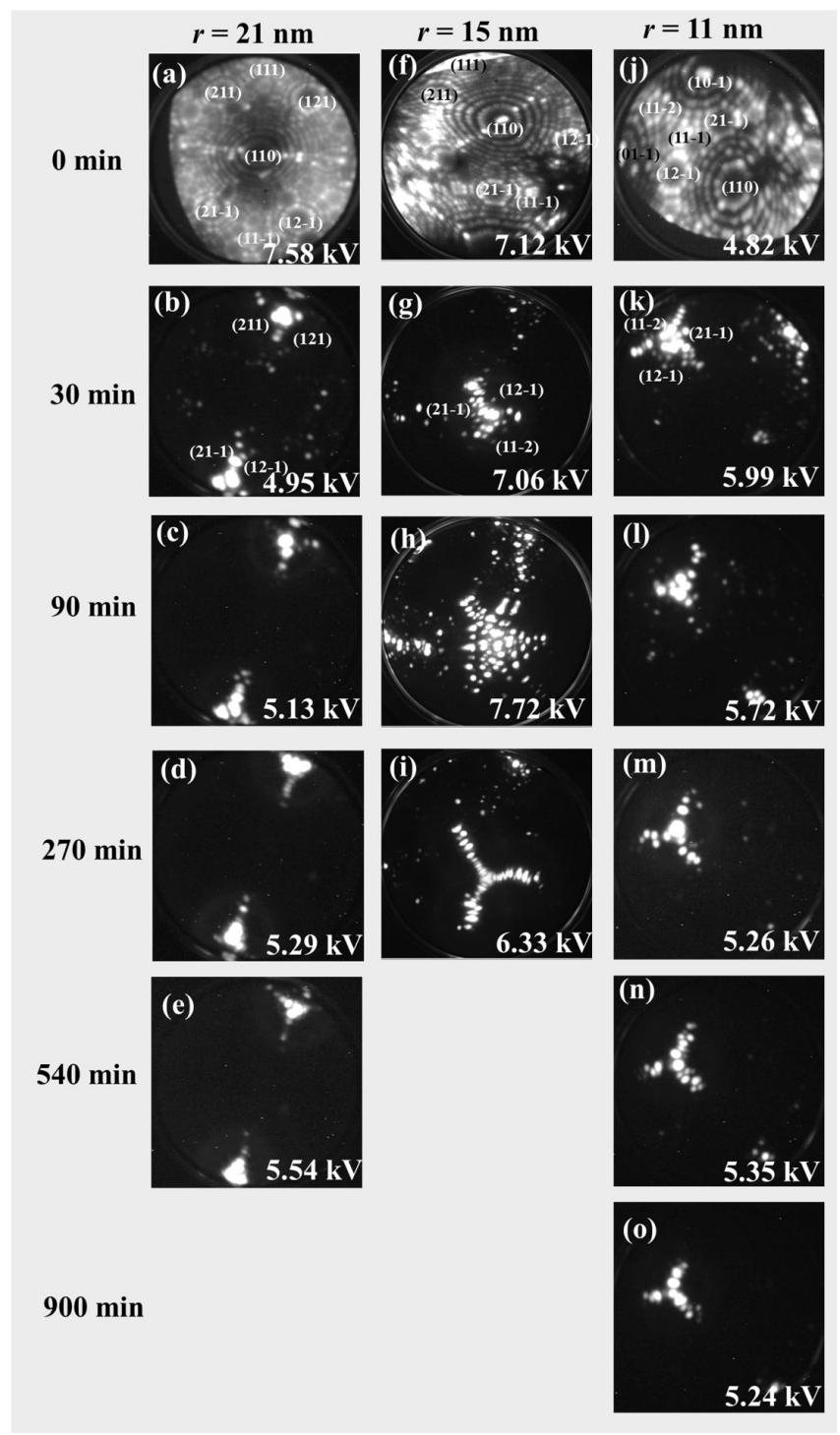

Figure 2: Structural evolution of Pd-coated W tips with different curvature radii as a function of annealing time. (a), (f), and (j) show He ion images of a clean $\mathrm{W}$ surface. The remaining images present the structural evolution of $\mathrm{W}$ tips, obtained by $\mathrm{Ne}$ ions. The values in the figure have the same meaning as those in Figure 1. show images of the growth of Pd-coated W tips with curvature radii with 15 and $11 \mathrm{~nm}$, respectively. For curvature radii of 21 and $15 \mathrm{~nm}$, nanopyramid growth is achieved by annealing for 540 and $270 \mathrm{~min}$, respectively. However, despite a prolonged annealing treatment at $1000 \mathrm{~K}$, a nanopyramid with a monoatomic chain ridge was not obtained for a curvature radius of $11 \mathrm{~nm}$. A comparison of the growth of the Au- and Pd-coated nanopyramids shows the following: (1) Both Au- and Pd-coated nanopyramids grew on W tips with curvature radii greater than $15 \mathrm{~nm}$. (2) The heating time required for nanopyramid formation on the Au-coated W tips tended to shorter than that for the Pd-coated W tips. (3) For a small curvature radius, the heating time was much longer than that for the larger curvature radii, contrary to our expectations. As described in the Introduction, previous studies have shown that nanopyramid growth does not occur on W substrate tips with curvature radii exceeding $200 \mathrm{~nm}$ [10-13]. Recently, Nakagawa reported that Pd-coated nanopyramids with monoatomic chain ridges cannot grow on $\mathrm{W}$ tips that have been mildly blunted by heating at $2000 \mathrm{~K}$, as determined by FIM [16]. The blunt $\mathrm{W}$ tip used in our experiment was blunted by field evaporation; thus, the curvature radii is smaller than that of the W tip used by Nakagawa. There is a gap between the size of $\mathrm{W}$ tips used in our present experiments and the size of $\mathrm{W}$ tips used in the previous study; thus, the formation of nanopyramids on W tips with intermediate curvature radii remains unknown. Nevertheless, the experimental results indicate that an ultrasharp tip is not necessary for nanopyramid formation, which is desirable for practical applications.

The difficulty encountered in the formation of nanopyramids on a $\mathrm{W}$ tip with a small curvature radius was unexpected. We suggest that the difficulty in nanopyramid growth is due to the ratio between the size of the nanopyramid base and that of the $\mathrm{W}$ substrate tip. According to previous scanning tunneling microscope research, the base length of a Pd-coated nanopyramid on a flat W surface ranges from $4.6 \pm 1.2$ to $6.6 \pm 1.7 \mathrm{~nm}$ for an annealing temperature of approximately $1000 \mathrm{~K} \mathrm{[17].} \mathrm{A} \mathrm{W} \mathrm{tip} \mathrm{with} \mathrm{a} \mathrm{cur-}$ vature radius of approximately $10 \mathrm{~nm}$ is small for constructing a nanopyramid. Assuming that there is an optimum size of $\mathrm{W}$ substrates for nanopyramid formation, the $\mathrm{W}$ tip should be blunted to an optimum curvature radius. This reasoning indicates that the nanopyramid formation process requires a substantial mass transport of $\mathrm{W}$ atoms to obtain a nanopyramid on a $\mathrm{W}$ tip with a small curvature radius. The mass transport depends on the surface self-diffusion coefficient $D_{\mathrm{s}}(T)$ of the $\mathrm{W}$ atoms and the anisotropy of the surface free energy [9]. Notably, there is a difference between the anisotropies of the surface free energy of binary systems consisting of $\mathrm{Au} / \mathrm{W}$ and $\mathrm{Pd} / \mathrm{W}$. Chen calculated the surface free energy, $\gamma$, of monolayer NM on various $\mathrm{W}$ planes by using the embedded atom method [18]. Specifically, $\gamma_{\mathrm{W}(111)}$, $\gamma_{\mathrm{Pd} / \mathrm{W}(211)}$, and $\gamma_{\mathrm{Au} / \mathrm{W}(211)}$ are 3510,2188 , and $1667 \mathrm{~mJ} \mathrm{~m}^{-2}$, respectively. Hence, we propose that the successful growth on a sharp W substrate for the Au case is associated with the anisotropic surface energies of a binary system consisting of 


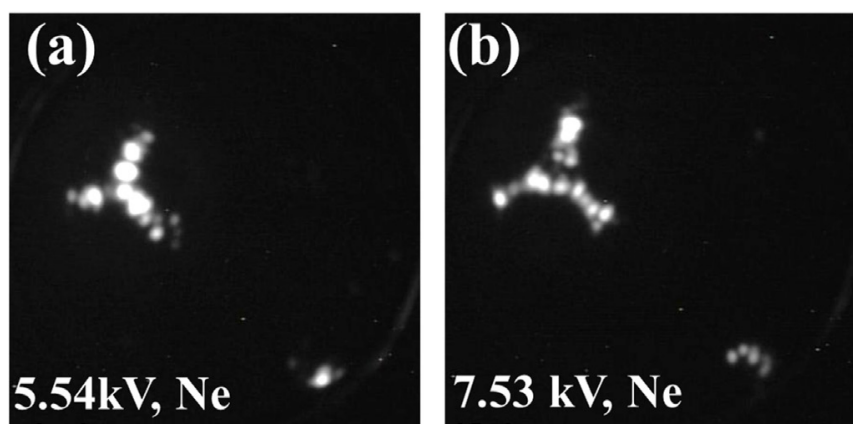

Figure 3: Structural change promoted by annealing at $1200 \mathrm{~K}$. (a) The conditions for this figure are the same as those for Figure 2(o). (b) Nanopyramid growth after 3 min of annealing at $1200 \mathrm{~K}$. Single-atom chain ridges, which include some defects in the nanopyramid, are formed.

an $\mathrm{Au}$ overlayer and a $\mathrm{W}$ substrate. To further confirm the insufficient mass transport of $\mathrm{W}$ atoms, we elevated the annealing temperature by a few hundred $\mathrm{K}$ for a short time (a few minutes) to promote surface self-diffusion of $\mathrm{W}$ atoms. Using the same treatment applied to the Pd-coated W tip presented in Figure 2(o), we present corresponding results in Figure 3. After annealing at $1200 \mathrm{~K}$ for $3 \mathrm{~min}$, we observed three-fold monoatomic chain ridges in the FIM image [Figure 3(b)]. These findings tentatively imply that the mass transport is promoted by high temperatures and accompanies the nanopyramid growth on a Pd-coated W tip with a curvature radius of $11 \mathrm{~nm}$. According to the phenomenalistic theory for blunting of the metal tip which was proposed by Barbour and co-works [19], the process of blunting occurs by step-down motion of atoms along steps of crystal plane which oriented tip apex. Comparing two images in Figure 3, the best image voltage in FIM observation has increased from 5.54 to $7.54 \mathrm{kV}$. The image voltage, $V$, and the curvature radius of the substrate $\mathrm{W}$ tip, $r$ related with the best image field for Ne, $F$ by $r=V / k F$, where $k$ is the geometric factor. Assuming that the geometric factor $k$ is maintained after the $1200 \mathrm{~K}$ annealing treatment, the increasing of best image voltage signified that the curvature radius of the $\mathrm{W}$ tip is increased and the blunting of the $\mathrm{W}$ tip is promoted by intense mass transport. We suppose that, when curvature radius of $\mathrm{W}$ substrate tip attains to sufficient size for construction of nanopyramid, the nanopyramid growth is completed. Therefore, the nanopyramid growth can be attributed to an increase in curvature radius. On the other hand, the extra-activation barrier for due to impurities, atomic defects, grain boundaries, or cluster aggregation on the $\mathrm{W}$ tip is quite conceivable for a mater delaying of growth of the nanopyramid. In such cases, it is uncontradictable that heating treatment at $1200 \mathrm{~K}$ drastically decreases the time of formative. We consider that such kinematic effect occurs in parallel with the blunting of the substrate $\mathrm{W}$ tip. The gain of elevating the annealing temperature by hundreds of Kelvins is considered with two ways: (1) overcomes the extra-activation barrier for the nanopyramid formation that caused by impurities, atomic defects, grain boundaries, or cluster ag- gregation on the W tip; (2) dulls curvature radius of the substrate $\mathrm{W}$ tip to suitable value for growth of the nanopyramid.

\section{CONCLUSIONS}

In summary, we investigated the formation process of Pdand Au-coated nanopyramids using $\mathrm{W}$ tips with different apex curvature radii as a substrate. By comparing the results for the Au- and Pd-coated W tips, we observed the following: (1) Both Au- and Pd-coated nanopyramids grew on W tips with curvature radii greater than $15 \mathrm{~nm}$. (2) The heating time required for nanopyramid formation of the Au-coated W tip tended to be shorter than that for the Pd-coated W tip. (3) The heating time for small curvature radii was much longer than that for larger curvature radii, in contrast to our expectations.

\section{Acknowledgments}

The authors would like to thank Professor Chuhei Oshima of Waseda University for suggestion of this work.

Note

This paper was presented at the 12th International Symposium on Atomic Level Characterizations for New Materials and Devices '19 (ALC '19), in conjunction with the 22nd International Conference on Secondary Ion Mass Spectrometry (SIMS-22), Miyako Messe, Kyoto, Japan, 20-25 October, 2019.

\section{References}

[1] H.-W. Fink, IBM J. Res. Dev. 30, 460 (1986).

[2] V. T. Binh and J. Marien, Surf. Sci. 202, L539 (1988).

[3] H. U. Müller, B. Völkel, M. Hofmann, Ch. Wöll, and M. Grunze, Ultramicroscopy 50, 57 (1993).

[4] K. Nagaoka, H. Fujii, K. Matsuda, M. Komaki, Y. Murata, C. Oshima, and T. Sakurai, Appl. Surf. Sci. 182, 12 (2001).

[5] T.-Y. Fu, L.-C. Cheng, C.-H. Nien, and T. T. Tsong, Phys. Rev. B 64,113401 (2001).

[6] H.-S. Kuo, I.-S. Hwang, T.-Y. Fu, J.-Y. Wu, C.-C. Chang, and T. T. Tsong, Nano Lett. 4, 2379 (2004).

[7] T. Nakagawa, E. Rokuta, H. Murata, H. Shimoyama, and C. Oshima, J. Vac. Sci. Technol. B 31, $02 B 105$ (2013).

[8] H. Asai, S. Kumagai, H. Murata, E. Rokuta, and C. Oshima, 2016 29th International Vacuum Nanoelectronics Conference (Vancouver, 2016) p. 1.

[9] M. Drechsler, in: Surface Mobilities on Solid Materials, edited by V. T. Binh (Springer US, New York, 1983) p. 405.

[10] A. Szczepkwowicz and A. Ciszewski, Surf. Sci. 515, 441 (2002).

[11] G. Antczak, T. E. Madey, M. Błaszczyszyn, and R. Błaszczyszyn, Vacuum 63, 43 (2001).

[12] G. Antczak, R. Błaszczyszyn, and T. E. Madey, Prog. Surf. Sci. 74, 81 (2003).

[13] A. Cetronio and J. P. Jones, Surf. Sci. 40, 227 (1973).

[14] E. W. Müller and T. T. Tsong, Field Ion Microscopy (American Elsevier Pub., New York, 1969).

[15] C. Kittel, Introduction to Solid State Physics, 8th ed. (Wiley Press, New York, 1975). 
[16] T. Nakagawa, E. Rokuta, H. Murata, H. Shimoyama, and C. Oshima, e-J. Surf. Sci. Nanotechnol. 10, 12 (2012).

[17] A. Szczepkwowicz, A. Ciszewski, R. Bryl, C. Oleksy, C.-H. Nien, Q. Wu, and T. E. Madey, Surf. Sci. 599, 55 (2005).

[18] S. P. Chen, Surf. Sci. 274, L619 (1992).

[19] J. P. Barbour, F. M. Charbonnier, W. W. Dolan, W. P. Dyke, E. E. Martin, and J. K. Trolan, Phys. Rev. 117, 1452 (1960).

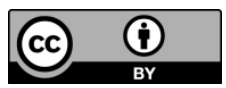

All articles published on e-J. Surf. Sci. Nanotechnol. are licensed under the Creative Commons Attribution 4.0 International (CC BY 4.0). You are free to copy and redistribute articles in any medium or format and also free to remix, transform, and build upon articles for any purpose (including a commercial use) as long as you give appropriate credit to the original source and provide a link to the Creative Commons (CC) license. If you modify the material, you must indicate changes in a proper way.

Published by The Japan Society of Vacuum and Surface Science 